\title{
Surface-Enhanced Raman Scattering Monitoring of \\ Oxidation States in Defect-Engineered Two-Dimensional Transition Metal Dichalcogenides
}

Xiangyu Hou, Qian Lin, Yunjia Wei, Qi Hao, Zhenhua Ni*, Teng Qiu*

School of Physics, Southeast University, Nanjing, 211189, P.R. China

Email: tqiu@seu.edu.cn, zhni@seu.edu.cn 

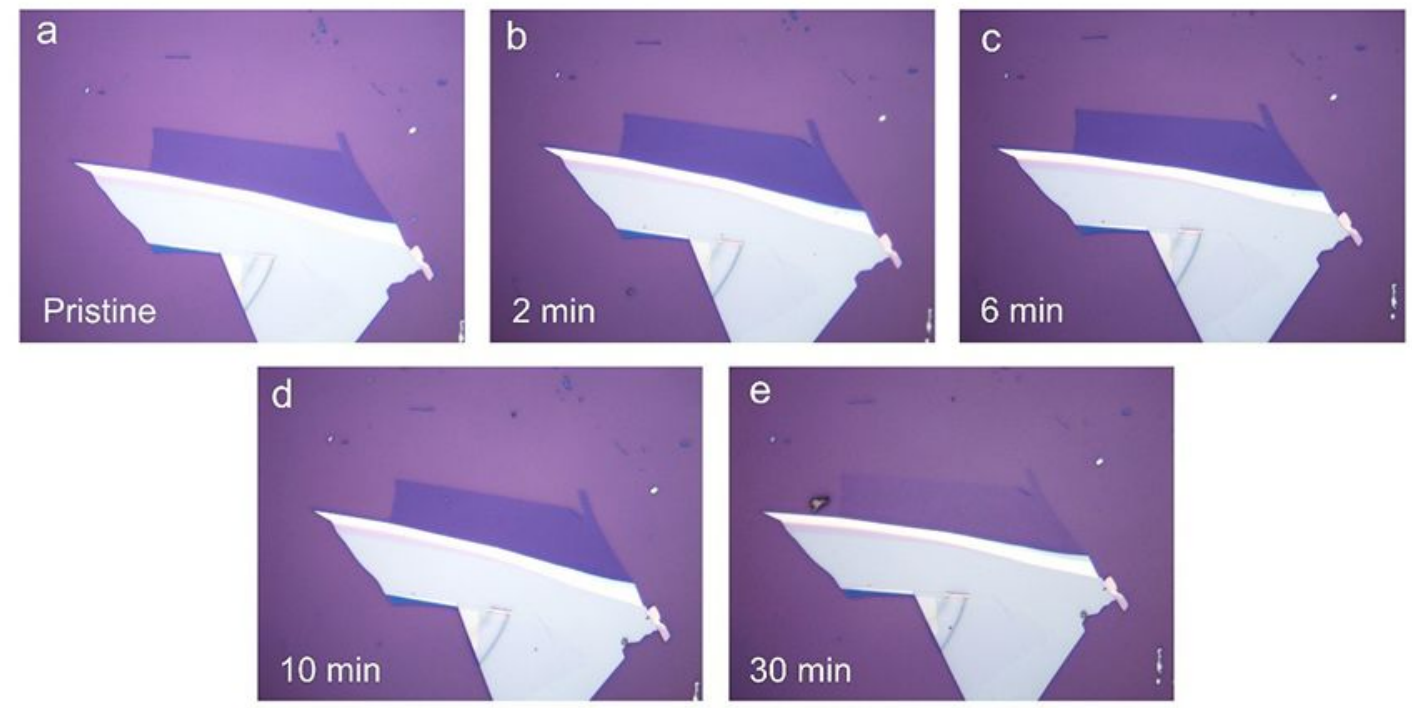

Figure S1. Optical micrographs of monolayer $\mathrm{MoS}_{2}$ with different UVO treatment times. These micrographs were measured by $100 \times$ objective lens. 


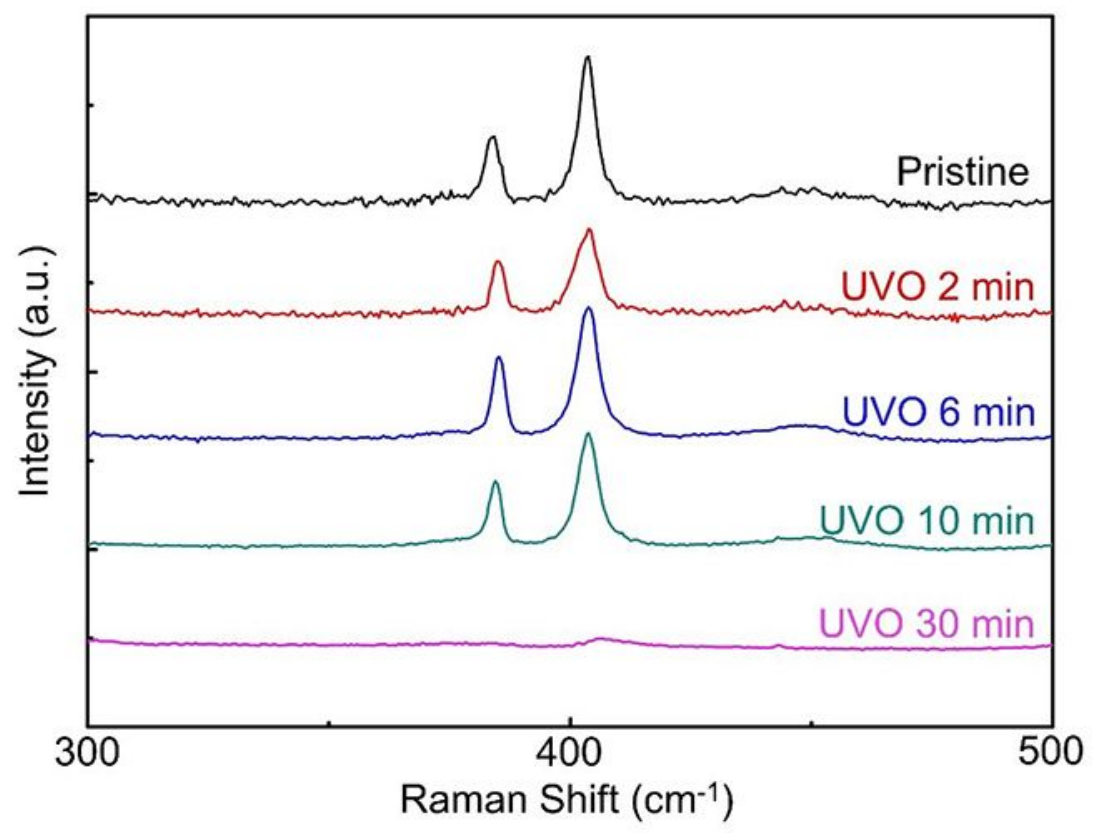

Figure S2. Raman spectra of monolayer $\mathrm{MoS}_{2}$ with different UVO treatment times. 

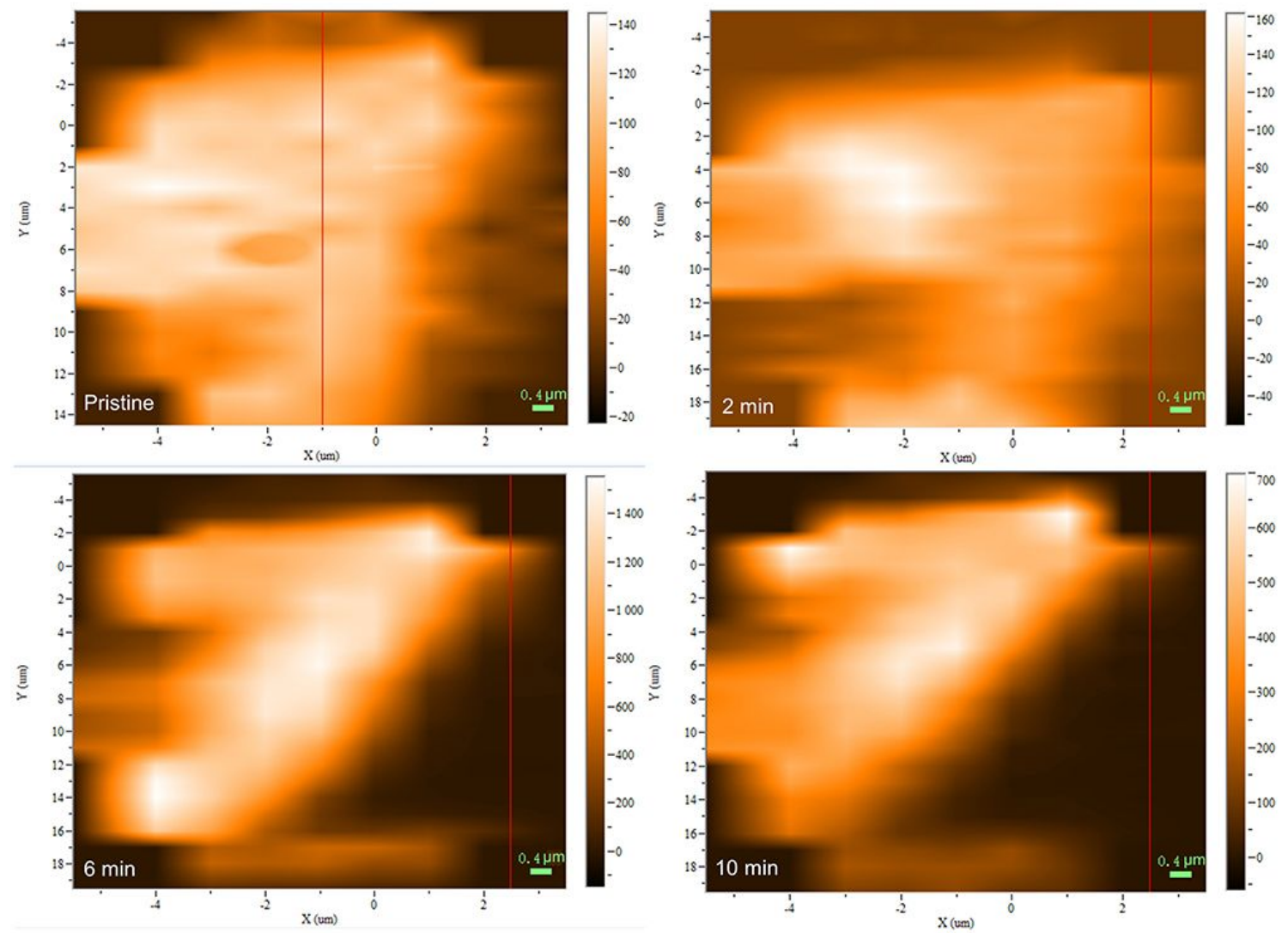

Figure S3. SERS maps of R6G on monolayer $\mathrm{MoS}_{2}$ under different UVO treatment times. The values of relative standard deviation on pristine and UVO-treated $\mathrm{MoS}_{2}$ are $9.05 \%, 14.99 \%, 12.85 \%$ and $14.94 \%$, respectively. 


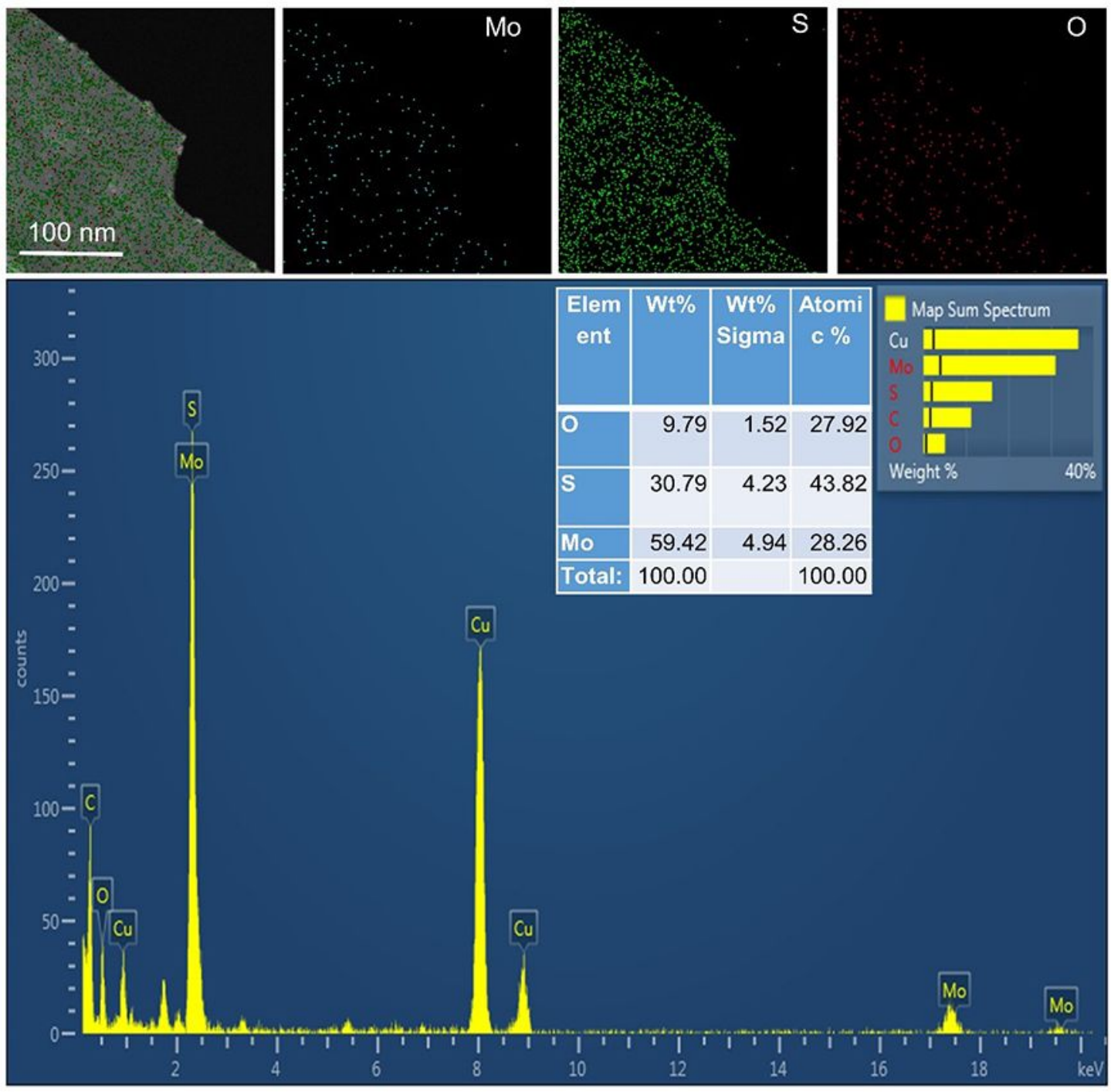

Figure S4. Elemental mappings (top) and energy-dispersive spectroscopy (bottom) of monolayer $\mathrm{MoS}_{2}$ at processing 6 minutes. 

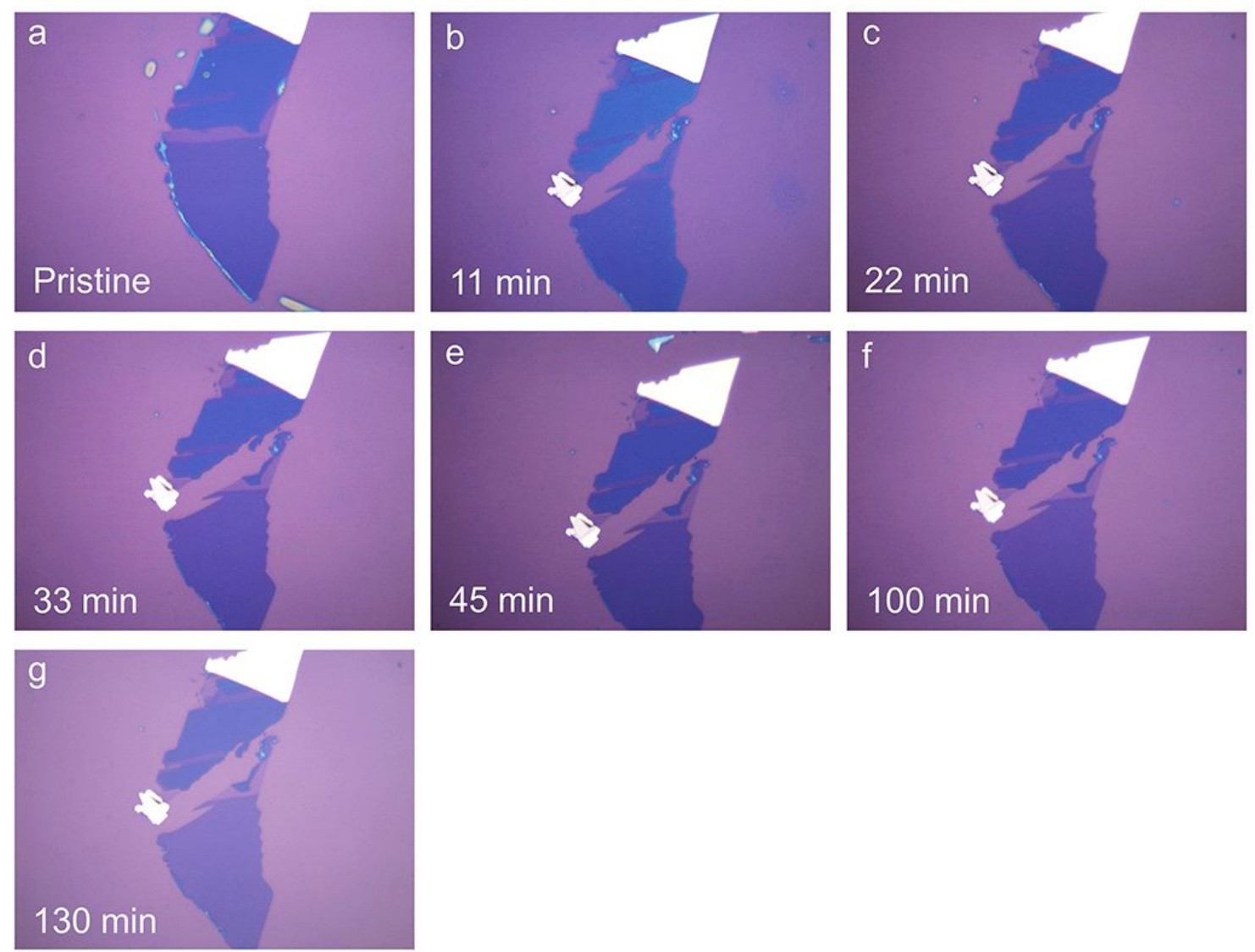

Figure S5. Optical micrographs of $2 \mathrm{~L}$ and $4 \mathrm{~L} \mathrm{MoS}_{2}$ with different UVO treatment times. These micrographs were measured by $100 \times$ objective lens. 


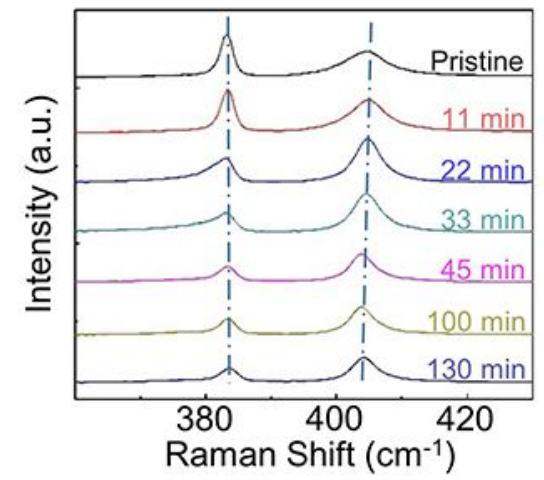

Figure S6. Raman spectra of $2 \mathrm{~L} \mathrm{MoS}_{2}$ with different UVO treatment times. 


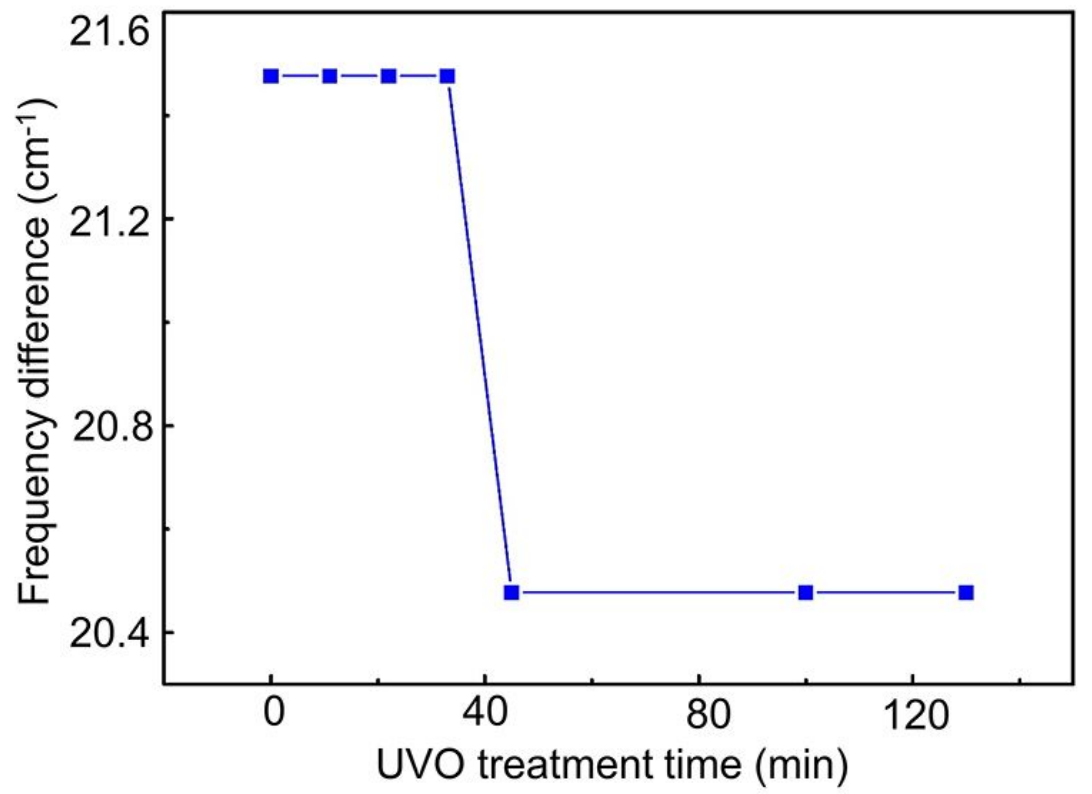

Figure S7. Frequency difference between $\mathrm{E}^{1}{ }_{2 \mathrm{~g}}$ and $\mathrm{A}_{1 \mathrm{~g}}$ bands of $2 \mathrm{~L} \mathrm{MoS}_{2}$ with different UVO treatment times. 


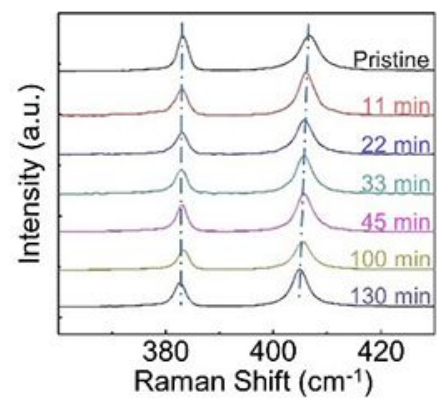

Figure S8. Raman spectra of $4 \mathrm{~L} \mathrm{MoS}_{2}$ with different UVO treatment times. 


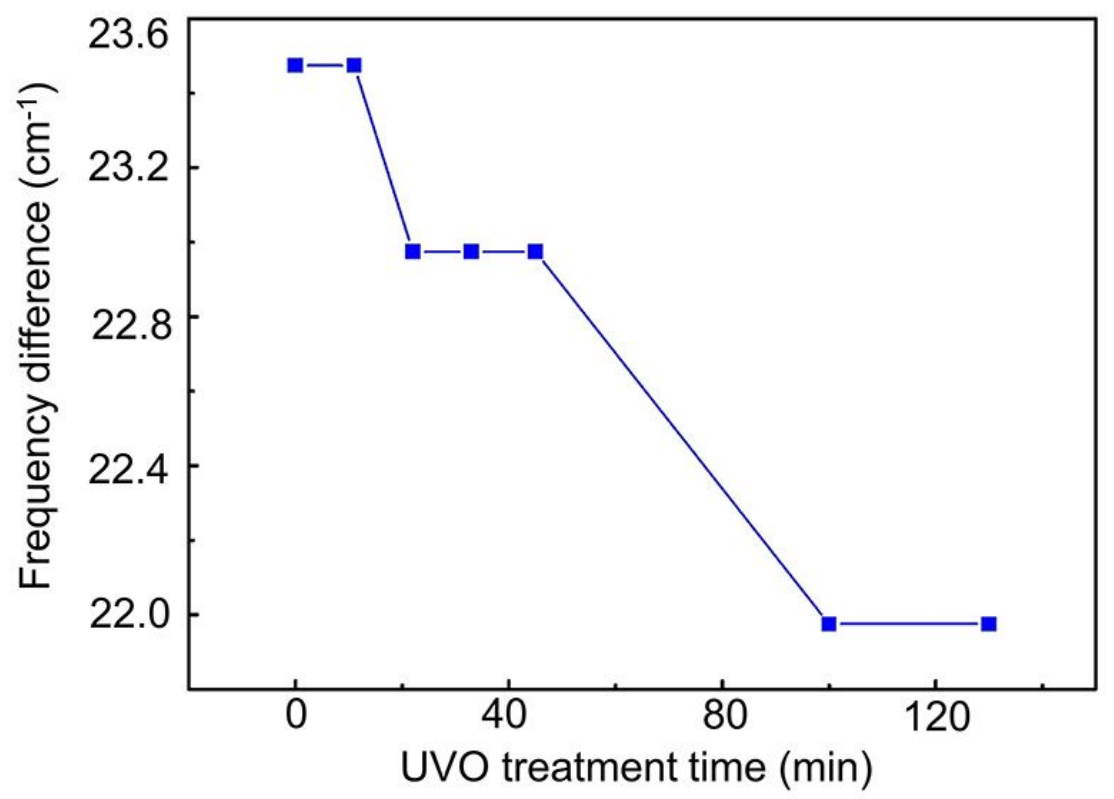

Figure S9. Frequency difference between $\mathrm{E}^{1}{ }_{2 \mathrm{~g}}$ and $\mathrm{A}_{1 \mathrm{~g}}$ bands of $4 \mathrm{~L} \mathrm{MoS}_{2}$ with different UVO treatment times. 

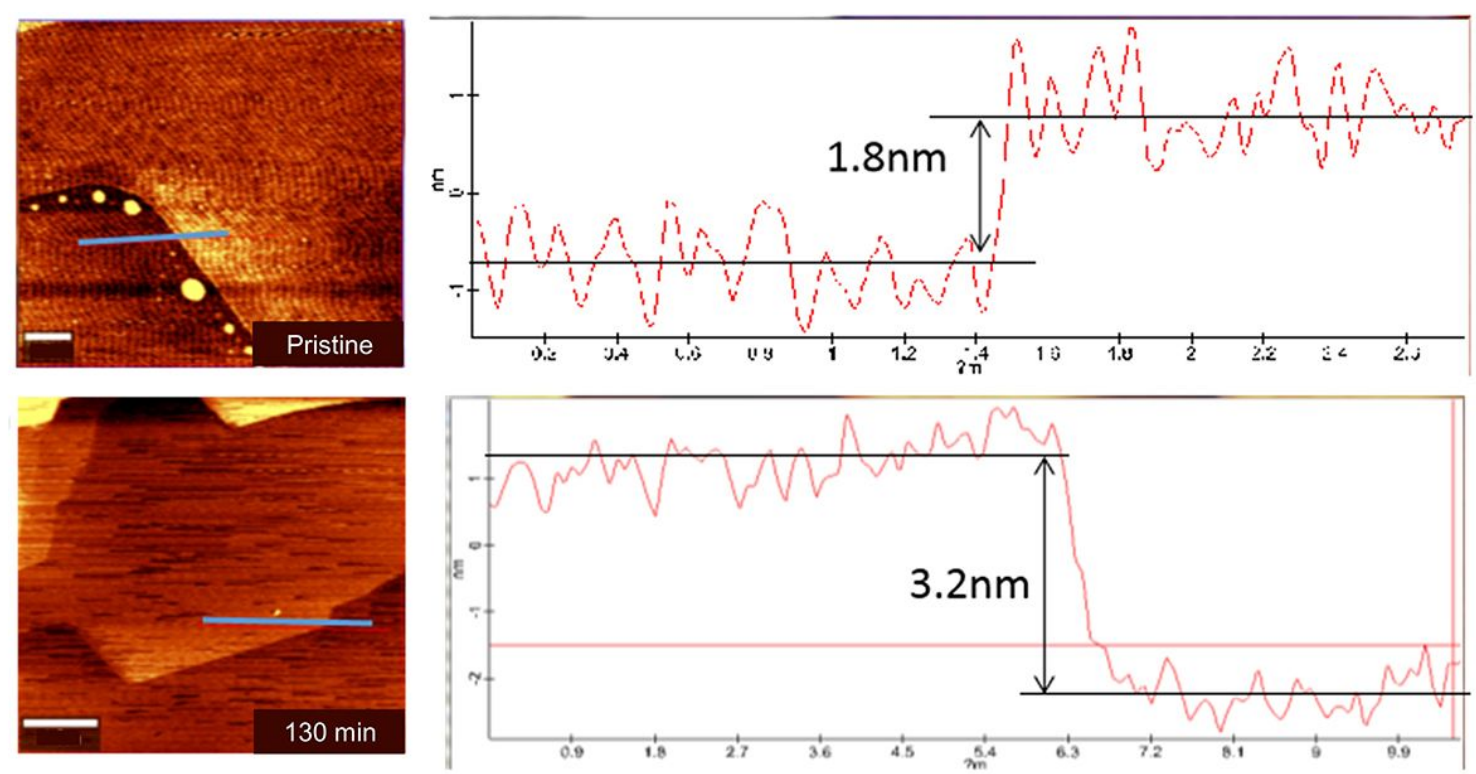

Figure S10. The atomic force microscopy images of pristine and UVO-treated $2 \mathrm{~L} \mathrm{MoS}$. 